\title{
Efficacy of oncolytic reovirus against human breast cancer cells
}

\author{
YOKO HATA $^{1}$, TSUYOSHI ETOH ${ }^{1}$, MASAFUMI INOMATA ${ }^{1}$, \\ NORIO SHIRAISHI ${ }^{1}$, AKIRA NISHIZONO ${ }^{2}$ and SEIGO KITANO ${ }^{1}$ \\ Departments of ${ }^{1}$ Surgery I and ${ }^{2}$ Microbiology, Oita University Faculty of Medicine, \\ 1-1 Hasama-machi, Yufu, Oita 879-5593, Japan
}

Received January 8, 2008; Accepted February 29, 2008

\begin{abstract}
Human reovirus can replicate and induce tumor cell lysis in several cancer types with an activated Ras signaling pathway. The aim of this study was to investigate the oncolytic effect of reovirus against breast cancer cells, and clarify the relationship between the susceptibility to reovirus and HER2 expression, which is associated with the Ras signaling pathway. Reovirus (serotype 3), 6 human breast cancer cell lines and normal mammary gland epithelial cell line were used in this study. The mRNA expression of HER2 receptor was examined by RT-PCR, and the protein of HER2 and activated Ras protein were examined by a Western blot analysis. In vitro, the cytopathic effects, viral protein synthesis, and cell viability on infection by reovirus were examined. Reovirus can infect all the 6 examined cancer cell lines, but the control cell line was not susceptible to the reovirus infection. The cytopathic effect appeared from day 1 after infection, and a 50\% or greater cytotolysis was demonstrated at day 7 after infection. The Ras activities in all examined cell lines were higher than those in the control cell line. No relationship was observed in the susceptibility to the reovirus and HER2 expression. The increased Ras activity itself, regardless of the HER2 expression, may therefore play an important role in the susceptibility to reovirus of breast cancer cells. As a result, breast cancer may thus become a candidate target for oncolytic reovirus therapy.
\end{abstract}

\section{Introduction}

Breast cancer is the leading malignant disease in women worldwide $(1,2)$. There are several treatment modalities such as surgery alone, or surgery combined with chemotherapy, endocrine therapy and radiotherapy against breast cancers, and several studies have shown good clinical outcomes of the patients treated with these treatments (3). Several

Correspondence to: Dr Yoko Hata, Department of Surgery I, Oita University Faculty of Medicine, 1-1 Hasama-machi, Yufu, Oita 879-5593, Japan

E-mail: yokohata@med.oita-u.ac.jp

Key words: reovirus, breast cancer, Ras activity, HER2 immunological approaches for breast cancer, including a monoclonal antibody specific for HER2 (Neu/ErbB-2), have been developed $(4,5)$. Although the survival rates of patients with breast cancer have been improving over the last two decade due to the development of the above therapeutic modalities (6), the existence of the patients resistant to these treatments has been reported. Therefore, a new approach against breast cancer to overcome this problem is needed.

Human reovirus is a unique oncolytic, non-enveloped virus containing 10 segments of double-stranded RNA as its genome. Reovirus always infects humans asymptomatically and it is occasionally isolated from the respiratory and gastrointestinal tract. Recent molecular studies have revealed that reovirus requires an activated Ras signaling pathway via either a direct ras mutation or independent pathways upstream of Ras such as epidermal growth factor receptor, HER2 or sos in order to be capable of propagating in cultured cells (7). Although ras mutations are rarely seen $(0-12 \%)$ in breast cancer (8), an aberrant Ras activation in breast cancer, because of an upregulation of the pathways downstream of Ras, has been reported and this activation was believed to be associated with cancer growth and progression (9). Norman et al demonstrated that reovirus infects breast cancer and has antitumor effects against human breast cancer (10).

The aim of this study was to investigate the oncolytic effect of reovirus against breast cancer cells and to clarify the relationship between susceptibility to reovirus and the expression of HER2, which has tyrosine kinase activity and is associated with the Ras signaling pathway.

\section{Materials and methods}

Cell lines and virus. Six human breast cancer cell lines including SK-BR-3, KPL4, MDA-MB-453, CRL1500, MCF7 and MDA-MB-231, and normal mammary gland epithelial cell line, Hs578Bst, as a control were used in this study. SKBR-3, CRL1500 and MCF7 were obtained from the Medical Cell Resources Center at the institute of Development, Aging and Cancer, Tohoku University, Japan. KPL4 was established and kindly provided by Dr Junichi Kurebayashi (Kawasaki Medical School, Okayama, Japan). MDA-MB-453 was obtained from the Riken Cell Bank (Tsukuba, Japan). MDAMB-231 and Hs578Bst were obtained from American Type Culture Collection (ATCC, Manassas, VA, USA). SK-BR-3, KPL4, CRL1500 and MCF7 were cultured in RPMI-1640 medium (Gibco BRL Life Technologies, Rockville, MD, 
USA) supplemented with $10 \%$ fetal bovine serum (FBS) (Gibco BRL Life Technologies) and antibiotics. MDA-MB453 and MDA-MB-231 were subcultured in L-15 medium (Gibco BRL Life Technologies) supplemented with 10\% FBS and antibiotics. Hs578Bst was cultured in Dulbecco's modified Eagle's medium (DMEM) (Gibco BRL Life Technologies) supplemented with $10 \%$ FBS, antibiotics and $30 \mathrm{ng} / \mathrm{ml}$ epidermal growth factor. Reovirus serotype 3 was kindly provided by Dr Kensuke Hirasawa (University of Calgary, Canada). Reovirus serotype 3 was propagated in L929 cells grown in suspension in DMEM containing 5\% FBS $(11,12)$.

Reverse transcription-PCR. Total-RNA was extracted from cells using the EZ1 RNA Cell Mini Kit (Qiagen, Hilden, Germany). The reverse transcription reaction was performed according to the previous description (13). The resulting cDNA $(1.5 \mu \mathrm{g})$ was amplified under the following conditions: $95^{\circ} \mathrm{C}$ for $35 \mathrm{sec}, 60^{\circ} \mathrm{C}$ for $35 \mathrm{sec}$ and $72^{\circ} \mathrm{C}$ for $35 \mathrm{sec}$. Primers for HER2 (forward, 5'-CGG GAG ATC CCT GAC CTG CTG GAA-3'; reverse, 5'-CTG CTG GGG TAC CAG ATA CTC CTC-3') were expected to yield a 300-bp amplified product after 33 cycle reaction (14). Primers for GAPDH (forward, 5'-GTC AAC GGA TTT GGT CTG GAT T-3'; reverse, 5'-AGT CTT CTG GGT GGC AGT GAT-3') were used for the amplification of 560-bp product after 28-cycle reaction.

Western blot analysis. Western blot analysis was performed as described previously (15). The membranes were incubated with a specific rabbit polyclonal antibody recognizing HER2 (used at dilution 1:1000) (Cell Signaling Technology, Beverly, MA, USA) and then were incubated with horseradish peroxidase-conjugated goat anti-rabbit secondary antibody (Cell Signaling Technology). The reacted membranes were subjected to an ECL kit WB detection system (Amersham Japan, Tokyo, Japan) and exposed to X-ray film.

Cytotoxity in breast cancer cell lines. To evaluate the cytotoxic effect of reovirus on breast cancer cells, each cell line was infected with reovirus at a multiplicity of infection (MOI) of 10 plaque forming units (PFUs)/cell. After the infection by reovirus, the morphologic changes of these cell lines were examined under microscopical observation magnified. Furthermore, on days 0-7 after infection with reovirus at an MOI of 10 PFUs/cell, the number of viable cells was counted by a hemocytometer using the trypan blue exclusion method.

To detect reovirus protein synthesis, each cell line with $80 \%$ confluent growth was infected with reovirus at a MOI of $10 \mathrm{PFU} / \mathrm{cell}$. At $24 \mathrm{~h}$ after infection, the medium was replaced with methionine free medium containing $10 \%$ FBS and $\left[{ }^{35} \mathrm{~S}\right]-$ methionine. After $3 \mathrm{~h}$ incubation, the medium was removed, and then the cells were washed with phosphate-buffered saline (PBS) and lysed. The labeled proteins were electrophoresed on $10 \%$ SDS-polacrylamide gel (SDS-PAGE) as described previously (16).

Measuring Ras activity. The Ras activity in breast cancer cells was examined by a ras activation assay kit (Upstate Biotechnology, Charlottesville, VA, USA) as described

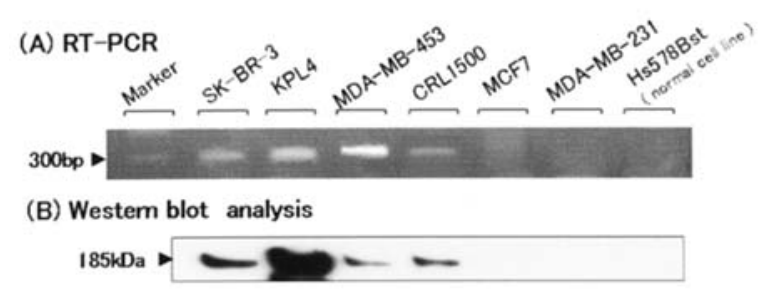

Figure 1. mRNA expression (A) and protein (B) of HER2 in the breast cancer cell lines. SK-BR-3, KPL4, MDA-MB-453 and CRL1500 cancer cell lines were positive for HER2, in contrast, MCF7, MDA- MB-231, Hs578Bst were negative for HER2.

previously (17). The samples were separated by SDS-PAGE, followed by electrobloting onto the PVDF membrane. A monoclonal anti-Ras antibody (clone RAS10) and horseradish peroxidase-conjugated goat anti-mouse secondary antibody were used to detect Ras protein. The amount of GTP bound Ras (activated Ras) was measured using the Raf1-Ras binding domain conjugated to agarose beads to pull down active Ras.

\section{Results}

Evaluation of HER2 expression in human breast cell lines. The mRNA expressions of HER2 receptor gene in human breast cell lines are shown in Fig. 1A. In 4 cancer cell lines SK-BR-3, KPL4, MDA-MB-453 and CRL1500, HER2 receptor gene were expressed as mRNA level. In contrast, no mRNA expressions of HER2 receptor gene were detected in the other cancer cell lines MCF7, MDA-MB-231 and Hs578Bst of the normal breast cell line.

Fig. 1B shows the expression of HER2 protein in human breast cell lines. HER2 protein was apparently expressed in 4 breast cancer cell lines SK-BR-3, KPL4, MDA-MB-453 and CRL1500, corresponding to the findings of RT-PCR analysis. In contrast, HER2 protein was not detected in MCF7, MDA-MB-231 and Hs578Bst.

In vitro oncolytic effect of reovirus. To evaluate the susceptibility of the human breast cell lines to reovirus, 6 human breast cell lines were infected at a MOI of $10 \mathrm{PFU} /$ cell. Regardless of the HER 2 expression in each cancer cell line, prominent morphological changes were seen in all cancer cell lines (Fig. 2). In contrast, the Hs578Bst of the normal breast cell line did not show apparent cytopathic effect (CPE) after infection and therefore this cell line was evaluated not to be susceptible to reovirus infection. In the cell viability test, all cancer cell lines showed efficiently cell death by reovirus infection within 1 week (Fig. 3). The CPE appeared from 1 day after infection, and $80 \%$ or greater cytotoxicity was demonstrated at day 7 after infection in all cell lines except for MDA-MB-231. The CPE in MDA-MB-231 was observed in $50 \%$ of the cells. Three major viral proteins $(\lambda, \mu, \sigma)$ were de novo synthesized in all cancer cell lines after a reovirus infection but not observed in the normal cell line, Hs578Bst (Fig. 4).

Detection of Ras activities in human breast cancer cell lines. Regardless of the HER2 expression in each cancer cell line, 


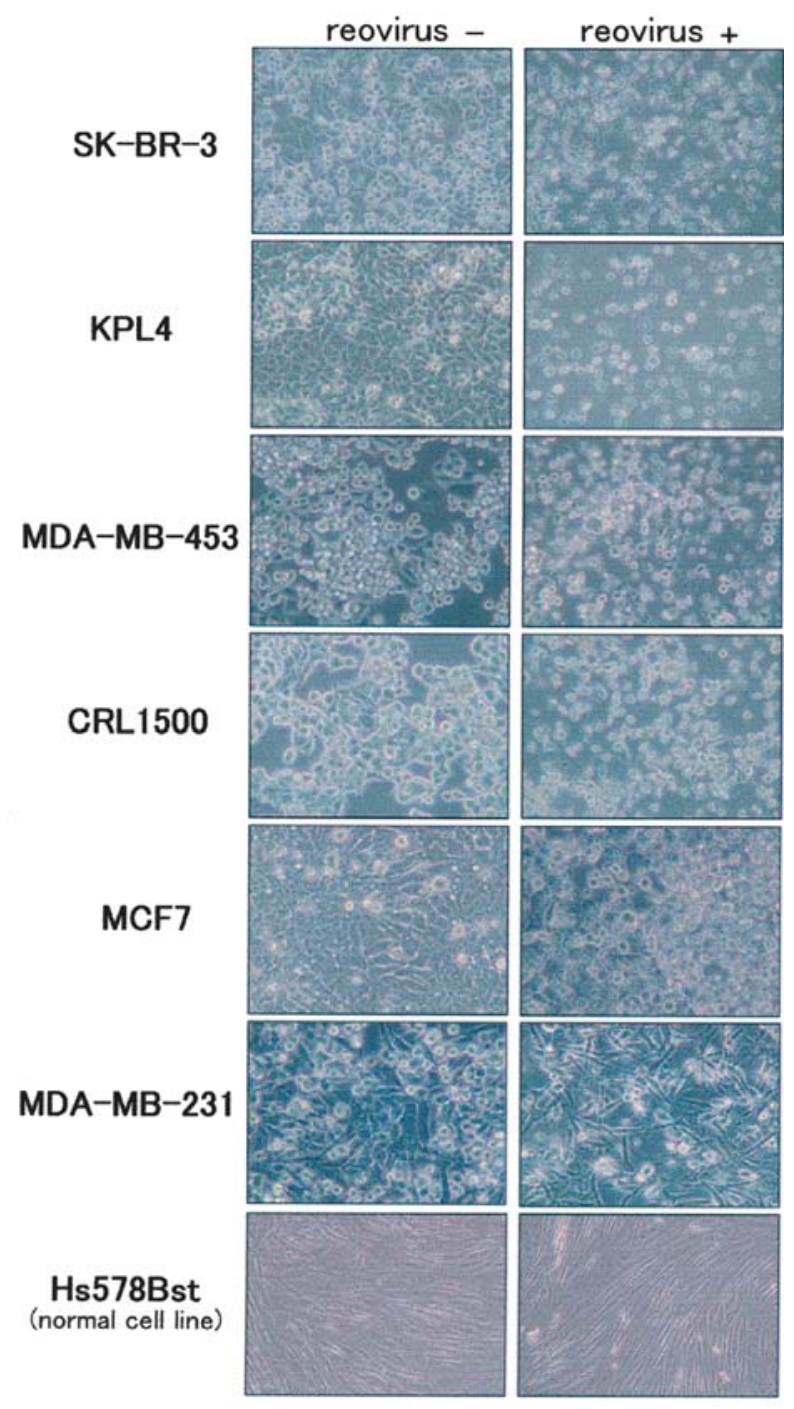

Figure 2. Morphological change in the breast cancer cell lines and normal breast cell line (Hs578Bst) at $72 \mathrm{~h}$ after infection of reovirus (MOI, $10 \mathrm{PFUs}$ / cell). Apparent CPE were observed in all cancer cell lines.

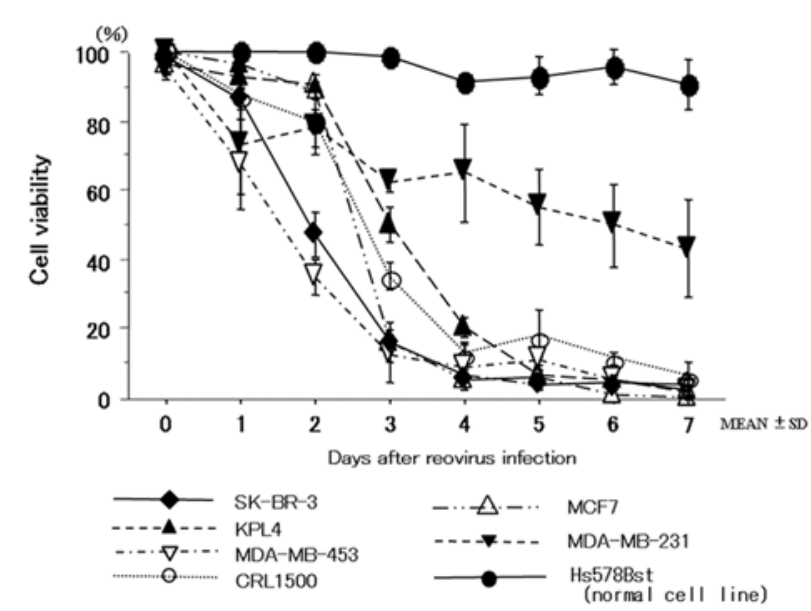

Figure 3. Cell viability in human breast cancer cell lines and normal breast cell line (Hs578Bst) after a reovirus infection. All human breast cancer cell lines were efficiently lysed by reovirus infection within 1 week.

the Ras activities in all cancer cell lines increased in comparison to those in the control cell line (Fig. 5).

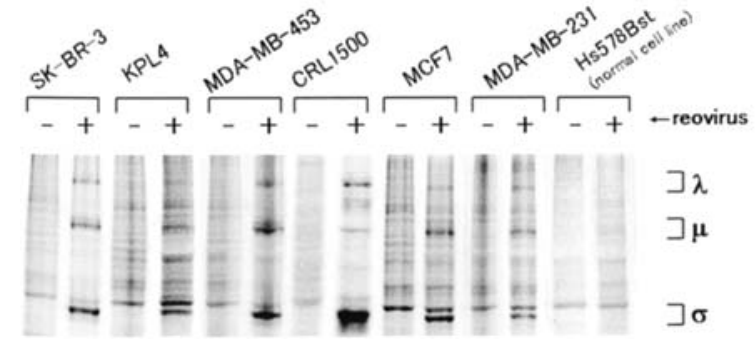

Figure 4. Reovirus protein synthesis in uninfected (-) and reovirus-infected (+) cell lines. The cells were infected with at a MOI of 10 PFUs per cell and, at the indicated times after infection, were pulse-labeled with $\left[{ }^{35} \mathrm{~S}\right]$-methionine for $3 \mathrm{~h}$. Right, the three size classes of reovirus protein, $\lambda, \mu$ and $\sigma$.

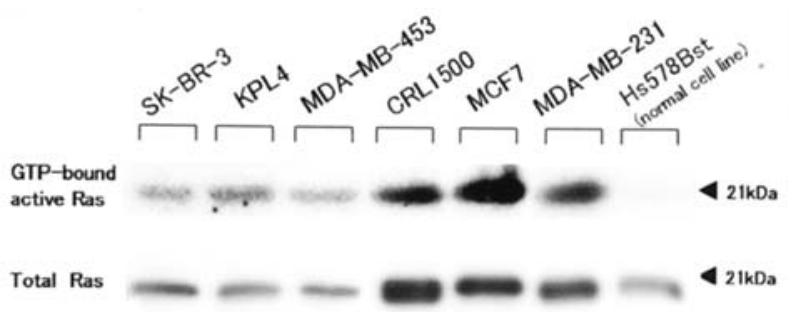

Figure 5. The Ras activity based on a Ras activation assay. The Ras activity increased in all breast cancer cell lines in comparison to the control cell line.

\section{Discussion}

Reovirus has recently been the focus of investigation as a novel oncolytic agent for cancer therapy based on targeting the activated Ras signaling pathway via direct Ras mutation or the independent pathways downstream of Ras $(18,19)$. Several experimental studies have thus demonstrated antitumor effects of reovirus against human colon cancer, ovarian cancer, pancreas cancer, glioma and lymphoid malignancies $(11,12,17,20,21)$.

In this study, we demonstrated that all examined breast cancer cell lines were susceptible to reovirus, while normal breast epithelial cells did not show susceptibility to the reovirus. This oncolytic effect of reovirus is related to the activated Ras activities regardless of the HER2 overexpression. These results suggest that reovirus can replicate in breast cancer cells via with an activated Ras signaling pathway and it might become as a new agent against breast cancer.

Several researchers have reported that the susceptibility of reovirus infection was affected by several cellular factors and/or signal pathways. Strong et al demonstrated that cells overexpressing the EGF receptor were highly susceptible to reovirus replication in comparison to parental control cells and also found that Ras promoted replication (7). Norman et al proposed that reovirus can be a novel therapy against breast cancer with a high Ras activity associated with the amplication of HER2 (10). In our study, the sensitivity of reovirus was observed in all breast cancer cell lines which we investigated, and these findings supported the proposal of Norman et al. The reason for promoting the infection of reovirus may be due to the fact that the Ras activities increased regardless of the HER2 expression, and these 
findings suggest that viral propagation in breast cancer cells might be strongly affected by an activated Ras pathway.

In the breast cancer cell lines that were used in this study, a ras mutation has only been reported in one cell line, MDAMB-231 (22), and the activated Ras in this cell line might be attributed to ras mutation, the examined breast cancer cell lines except MDA-MB-231 and MCF7 have HER2 expression, which affects Ras activity. Therefore, breast cancer may be a good target for reovirus, because a high frequency of activated Ras is often observed. It is necessary to clarify the mechanism of the Ras activity in breast cancer cells. Furthermore, there is a need to investigate and analyze the expression of other factors, such as sos, Raf, PI3K and RalGEF (23).

In clinical setting, the following prognostic factors of breast cancer have been identified: tumor size, age, lymph node metastasis, hormone receptor and HER2 (24). HER2 overexpression has been correlated with lymph node metastasis, thus resulting in significantly lower overall survival rates (25). The overall survival rates of the 1-3 node-positive patients with HER 2 positivity at 5 years were $46 \pm 14 \%$, while those with HER2 negative tumors were $81 \pm 4 \%$ (25). Our data suggest that reovirus might be a powerful tool against breast cancer being closely associated with a HER2 overexpression and activated Ras. From the view point of clinical application, breast cancer is superficially visible on the surface of the chest wall and it can be easily identified by sonography. Therefore, the injection of reovirus into the breast tumor may be easily performed with minimal invasion and side effects. However, further examinations, including the antitumor effect using small animal model, are necessary.

In conclusion, the susceptibility of reovirus to all breast cancer cell lines used in this study was observed and this oncolytic effect of reovirus was related to the activated Ras. Although the HER2 expression may affect the Ras activity, it was not a predictor of susceptibility to reovirus. Since activated Ras was recognized in many of breast cancer cells, breast cancer may be a good candidate for reovirus therapy.

\section{Acknowledgements}

This study was supported by the Ministry of Education, Culture and Science of Japan (grant no. 16591331).

\section{References}

1. Parkin DM, Bray F, Ferlay J and Pisani P: Global cancer statistics, 2002. CA Cancer J Clin 55: 74-108, 2005.

2. Veronesi U, Boyle P, Goldhirsch A, et al: Breast cancer. Lancet 365: 1727-1741, 2005.

3. Namer M, Fargeot P, Roche H, et al: French Adjuvant Study Group. Improved disease-free survival with epirubicin-based chemoendocrine adjuvant therapy compared with tamoxifen alone in one to three node-positive, estrogen-receptor-positive, post-menopausal breast cancer patients: results of French Adjuvant Study Group 02 and 07 trials. Ann Oncol 17: 65-73, 2006.
4. Papazisis KT, Habeshaw T and Miles DW: Herceptin-EAP Study Group. Safety and efficacy of the combination of trastuzumab with docetaxel for HER2-positive women with advanced breast cancer. Int J Clin Pract 58: 581-586, 2004.

5. Cobleigh MA, Vogel CL, Tripathy D, et al: Multinational study of the efficacy and safety of humanized anti-HER 2 monoclonal antibody in women who have HER2-overexpressing metastatic breast cancer that has progressed after chemotherapy for metastatic disease. J Clin Oncol 17: 2639-2648, 1999.

6. Mina L and Slegde GJ: Twenty years of systemic therapy for breast cancer. Oncology 20: 25-32, 2006.

7. Strong JE, Coffey MC, Tang D, Sabinin P and Lee PW: The molecular basis of viral oncolysis: usurpation of the Ras signaling pathway by reovirus. EMBO J 17: 3351-3362, 1998.

8. Kiaris $\mathrm{H}$ and Spandidos DA: Mutation of ras genes in human tumours (Review). Int J Oncol 7: 413-421, 1995.

9. Clark GJ and Der CJ: Aberrant function of the Ras signal transduction pathway in human breast cancer. Breast Cancer Res Treat 35: 133-144, 1995.

10. Norman KL, Coffey MC, Hirasawa K, et al: Reovirus oncolysis of human breast cancer. Hum Gene Ther 13: 641-652, 2002.

11. Yada K, Shibata K, Matsumoto T, Ohta M, Yokoyama S and Kitano S: Protease-activated receptor-2 regulates cell proliferation and enhances cyclooxygenase- 2 mRNA expression in human pancreatic cancer cells. J Surg Oncol 89: 79-85, 2005.

12. Krahn G, Leiter U, Kaskel P, et al: Coexpression patterns of EGFR, HER2, HER3 and HER4 in non-melanoma skin cancer. Eur J Cancer 37: 251-259, 2001.

13. Matsui $\mathrm{Y}$, Inomata M, Tojigamori M, et al: Suppression of tumor growth in human gastric cancer with HER2 overexpression by an anti-HER 2 antibody in a murine model. Int $\mathrm{J}$ Oncol 27: 681-685, 2005.

14. Lee PW, Hayes EC and Joklik WK: Protein sigma 1 is the reovirus cell attachment protein. Virology 108: 156-163, 1981.

15. Hirasawa K, Nishikawa SG, Norman KL, et al: Oncolytic reovirus against ovarian and colon cancer. Cancer Res 62: 1696-1701, 2002.

16. Coffey MC, Strong JE, Forsyth PA and Lee PW: Reovirus therapy of tumors with activated Ras pathway. Science 282: 1332-1334, 1998

17. Norman KL and Lee PW: Reovirus as a novel oncolytic agent. J Clin Invest 105: 1035-1038, 2000.

18. Wilcox ME, Yang W, Senger D, et al: Reovirus as an oncolytic agent against experimental human malignant gliomas. J Natl Cancer Inst 93: 903-912, 2001.

19. Alain T, Hirasawa K, Pon KJ, et al: Reovirus therapy of lymphoid malignancies. Blood 100: 4146-4153, 2002.

20. Etoh T, Himeno Y, Matsumoto T, et al: Oncolytic viral therapy for human pancreatic cancer cells by reovirus. Clin Cancer Res 9: 1218-1223, 2003.

21. Himeno Y, Etoh T, Matsumoto T, et al: Efficacy of oncolytic reovirus against liver metastasis from pancreatic cancer in immunocompetent models. Int J Oncol 27: 901-906, 2005.

22. Kozma SC, Bogaard ME, Buser K, et al: The human c-Kirsten ras gene is activated by a novel mutation in codon 13 in the breast carcinoma cell line MDA-MB231. Nucleic Acids Res 15: 5963-5971, 1987

23. Shmulevitz M, Marcato P and Lee PW: Unshackling the links between reovirus oncolysis, Ras signaling, translational control and cancer. Oncogene 24: 7720-7728, 2005.

24. Goldhirsch A, Glick JH, Gelber RD, et al: Meeting highlights: international expert consensus on the primary therapy of early breast cancer 2005. Ann Oncol 16: 1569-1583, 2005.

25. Tandon AK, Clark GM, Charmness GC, et al: HER-2/neu oncogene protein and prognosis in breast cancer. J Clin Oncol 7: 1120-1128, 1989. 